\title{
Pathological complete response after percutaneous isolated hepatic perfusion in hepatocellular carcinoma with portal vein tumor thrombosis: a case report
}

\author{
Keisuke Arai", Takumi Fukumoto, Motofumi Tanaka, Kaori Kuramitsu, Masahiro Kido, Hisoka Kinoshita, \\ Taku Matsumoto, Hirochika Toyama, Sadaki Asari, Tadahiro Goto, Tetsuo Ajiki and Yonson Ku
}

\begin{abstract}
Background: Although the effectiveness of perioperative adjuvant therapy in the treatment of hepatocellular carcinoma (HCC) has been investigated, the efficacy of preoperative therapy is unclear. Herein, we report a case of pathological complete response after percutaneous isolated hepatic perfusion (PIHP) for HCC involving portal vein tumor thrombosis (PVTT).

Case presentation: A 77-year-old woman was referred to our institute with a liver mass detected on a routine health screening. Computed tomography revealed a $28 \times 25 \mathrm{~mm} \mathrm{HCC}$ in the left lobe of the liver and a tumor thrombus in the left and right portal branches (T4NOMO, stage IVA). The patient received a single dose of preoperative PIHP with doxorubicin plus mitomycin C, without severe toxicity. After the chemotherapy, she underwent extended left hepatic lobectomy and thrombectomy of the PVTT. No cancer cells were detected during histopathological analysis, indicating pathological complete response. She remained relapse-free 12 months after the surgery.

Conclusions: We experienced a case of pathological complete response after preoperative PIHP with doxorubicin plus mitomycin C for HCC involving PVTT.
\end{abstract}

Keywords: Hepatocellular carcinoma, Portal vein tumor thrombosis, Percutaneous isolated hepatic perfusion, Preoperative chemotherapy

\section{Background}

Hepatocellular carcinoma (HCC) is the sixth most common cancer and third leading cause of cancer-related deaths worldwide [1]. Surgical resection is the most promising treatment for long-term survival although only $10-37 \%$ of the cases with HCC are eligible for surgical resection because of the extent of disease or cirrhosis-related liver dysfunction [2]. Portal vein tumor thrombosis (PVTT) is a common complication of HCC associated with a poor prognosis [3]. For the treatment of HCC involving PVTT, according to Japanese Clinical

\footnotetext{
* Correspondence: karai51@outlook.jp

Division of Hepato-Biliary-Pancreatic Surgery, Department of Surgery,

Graduate School of Medicine, Kobe University, 7-5-2, Kusunoki-cho, Chuo-ku, Kobe, Hyogo 650-0017, Japan
}

Practice Guidelines for Hepatocellular Carcinoma 2013, liver resection may be selected for patients with liver damage of grade A along with three or less HCC [4]. However, the use of curative surgical resection is controversial because it is associated with a high recurrence rate [5-9]. Therefore, effective multimodality treatment approaches should be explored to improve the overall and survival outcomes. Percutaneous isolated hepatic perfusion (PIHP) is a form of high-dose regional chemotherapy that enables the administration of cytotoxic agents at a dose of up to 10 times the maximally tolerated dose, while reducing exposure of the entire body to the major adverse effects of the therapeutic agents [10]. We showed in our previous studies that PIHP is safe and effective in the treatment of local tumors, even in patients with macroscopic PVTT 
$[11,12]$. In order to avoid further tumor cell dissemination and reduce the stage of the tumor before surgery, we tested the efficacy of administering PIHP before surgery. Herein, we report a case of HCC involving PVTT for which a pathological complete response was achieved by the administration of PIHP with doxorubicin plus mitomycin $\mathrm{C}$ before surgery.

\section{Case presentation}

A 77-year-old woman who was diagnosed with chronic hepatitis $C$ at the age of 50 years was referred to a local hospital for follow-up. Abdominal ultrasonography showed a tumor in the left lobe of the liver. She was referred to our department for further examination and treatment. Laboratory data were as follows: platelet count $126 \mathrm{~K} / \mathrm{uL}$, total bilirubin $0.8 \mathrm{mg} / \mathrm{dL}$, aspartate aminotransferase (AST) $176 \mathrm{U} / \mathrm{L}$, alanine aminotransferase (ALT) $157 \mathrm{U} / \mathrm{L}$, albumin $4.2 \mathrm{~g} / \mathrm{dL}$, prothrombin time $90.6 \%$, indocyanine green 15-min retention test (ICG R15) $19.7 \%$, $\alpha$-fetoprotein (AFP) $16 \mathrm{ng} / \mathrm{mL}$, protein induced by vitamin $\mathrm{K}$ absence or antagonist II (PIVKA-II) $2873 \mathrm{mAU} / \mathrm{mL}$, hepatitis $\mathrm{C}$ virus $(\mathrm{HCV})$ Ab positive, $\mathrm{HCV}$ RNA 6.54 $\log \mathrm{IU} / \mathrm{mL}$. The liver damage was grade A and Child-Pugh classification was class A. Contrast-enhanced computed tomography (CT) showed a $28 \times 25 \mathrm{~mm}$ tumor in segment 4, with a tumor thrombus in the left portal branch reaching the right portal branch, both of which were enhanced in the arterial phase and washed out in the equilibrium phase (Fig. 1). The patient was diagnosed with clinical stage T4 (Vp4) bNOM0, stage IVA HCC involving
PVTT, according to the tumor-node-metastasis classification of the Liver Cancer Study Group in Japan (6th edition) [13], which indicated poor prognosis when treated with surgery only. After providing informed consent, the patient decided to receive PIHP before surgery. She received continuous hepatic arterial infusion (HAI) of $100 \mathrm{mg} / \mathrm{m}^{2}$ doxorubicin plus $30 \mathrm{mg} / \mathrm{m}^{2}$ mitomycin $\mathrm{C}$ for $30 \mathrm{~min}$ (Fig. 2). She recovered from a single dose of PIHP without severe toxicity, except a reduction in the grade 4 neutrophil counts (neutrophil count 200/ $\mathrm{L}$ ) that was treated with recombinant G-CSF (CTCAE version 4.0) [14] on the 12th day after PIHP. The serum level of AST and ALT rose up to $516 \mathrm{U} / \mathrm{L}$ and $242 \mathrm{U} / \mathrm{L}$ on the 4th day after PIHP, respectively. CT showed reduction of tumor volume and no enhancement of the PVTT after PIHP (Fig. 3). The efficacy of chemotherapy was determined to be treatment efficacy (TE) 3 according to the criteria of the Liver Cancer Study Group in Japan (6th edition) [13] and based on the partial response achieved. The clinical stage after PIHP was determined to be T4N0M0, stage IVA. The serum levels of AFP and PIVKA-II dramatically declined to $16 \mathrm{ng} / \mathrm{mL}$ and $21 \mathrm{mAU} / \mathrm{mL}$, respectively (Fig. 4). Subsequently, the neutrophil increased to 2400/ $\mu \mathrm{L}$, and the serum levels of AST/ALT improved to 36/ $61 \mathrm{U} / \mathrm{L}$. The patient underwent extended left hepatic lobectomy and PVTT thrombectomy 6 weeks after PIHP. The operative findings revealed the extent of PVTT to be from the left and right portal branches to the portal trunk. Because the PVTT did not adhere to the portal vein wall, which would have led to a combined resection, she
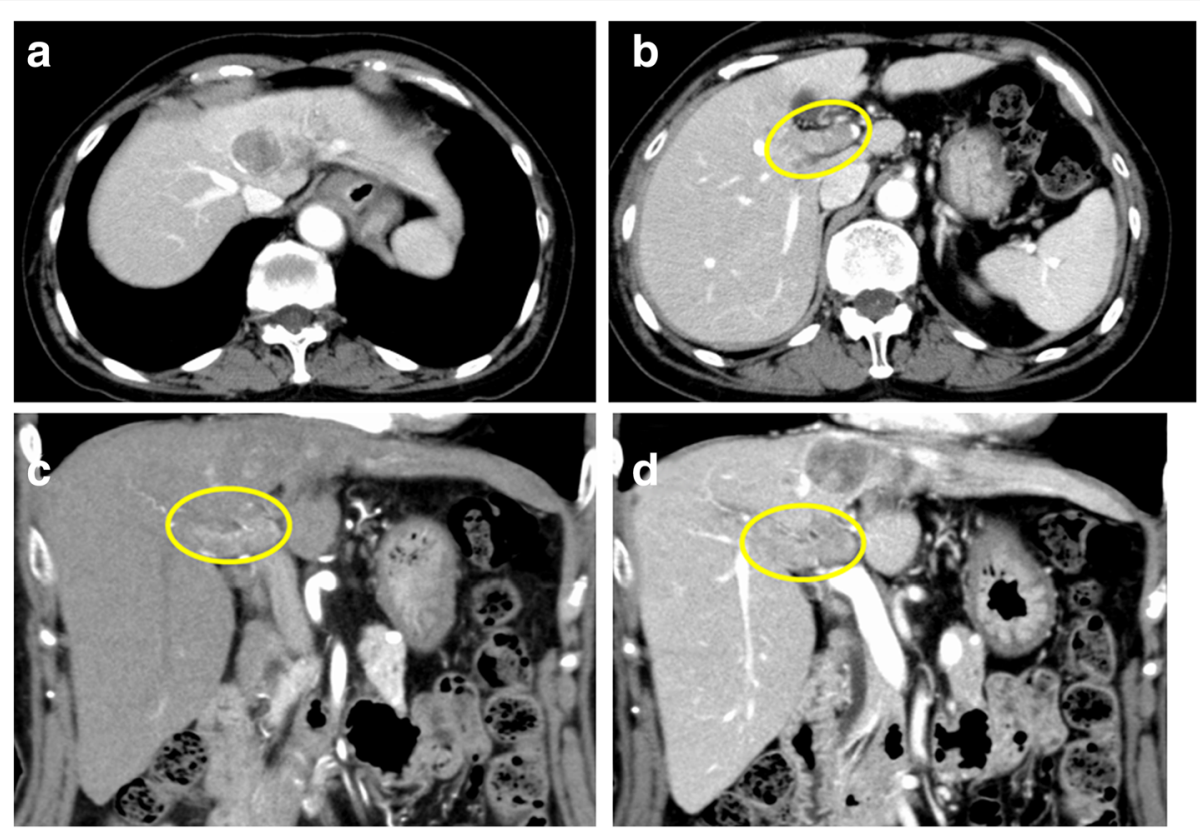

Fig. 1 Contrast-enhanced CT before preoperative PIHP: CT showing a $28 \times 25 \mathrm{~mm}$ HCC in segment 4 (a) and PVTT (yellow circle) in the left portal branch reaching the right portal (b). PVTT was enhanced in the arterial phase (c) and washed out in the equilibrium phase (d) 


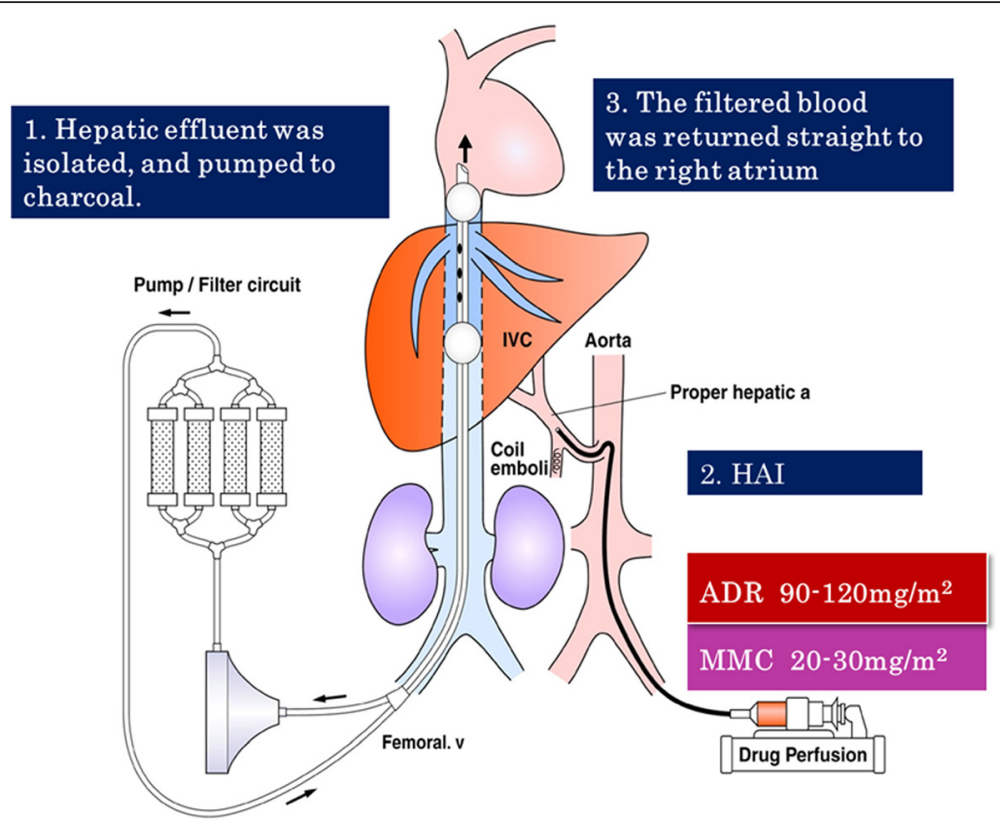

Fig. 2 System of PIHP: The specially designed 4-lumen/2-balloon catheter was introduced into the retrohepatic inferior vena cava through the femoral vein. The hepatic effluent was isolated by balloon inflation and pumped to charcoal filters via the fenestrations of one major catheter lumen. The filtered blood was returned straight to the right atrium through the other major lumen with an opening at the distal tip of the catheter. The patient received a 30-min continuous HAl of $100 \mathrm{mg} / \mathrm{m}^{2}$ doxorubicin plus $30 \mathrm{mg} / \mathrm{m}^{2}$ mitomycin C. At the end of HAl, extracorporeal drug filtration was maintained at least for $10 \mathrm{~min}$, and the 4-lumen/2-balloon catheter was removed

underwent thrombectomy with the back flow perfusion method and closure of the stump through a running suture of the portal vein with radical intent.

Histological examination of the resected specimen revealed complete necrosis without any viable cancer cells in the primary tumor and PVTT (Fig. 5) and we further showed that treatment efficacy 4 and pathological complete response were achieved. The patient recovered uneventfully after surgery and remained relapse-free 12 months after the surgery, without any postoperative chemotherapy.

\section{Discussion}

The optimal treatment method for patients with HCC involving PVTT in the portal bifurcation or the main trunk remains controversial [15]. PVTT represents one of the most important prognostic factors in patients with HCC.
Most patients with HCC involving extensive PVTT have multiple intrahepatic metastases at the time of diagnosis and they are considered poor surgical candidates. If left untreated, patients with HCC involving PVTT have a median overall survival (OS) of $2-4$ months $[16,17]$. PVTT is also a causative factor for intrahepatic tumor dissemination, which primarily accounts for the poor prognosis in this group of patients $[18,19]$. The most common staging system for HCC employed in American and European centers, the Barcelona Clinic Liver Cancer (BCLC) system, recommends against surgical resection in cases involving PVTT [20]. In a previous series of patients who underwent hepatectomy and thrombectomy for HCC involving PVTT in the main portal trunk, the 1- and 3-year OS rates were 25 and $4 \%$, respectively, and the disease-free survival rates were $3 \%$ and $0 \%$, respectively [21]. However, surgical resection is considered the only curative

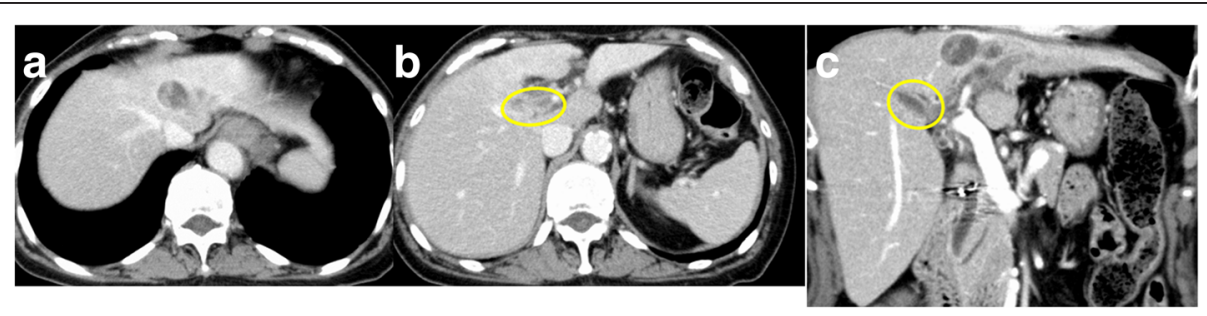

Fig. 3 Contrast-enhanced CT after preoperative PIHP: CT showing the reduction of the main tumor volume (a) and no enhancement of the PVTT (yellow circle) after PIHP (b, c) 


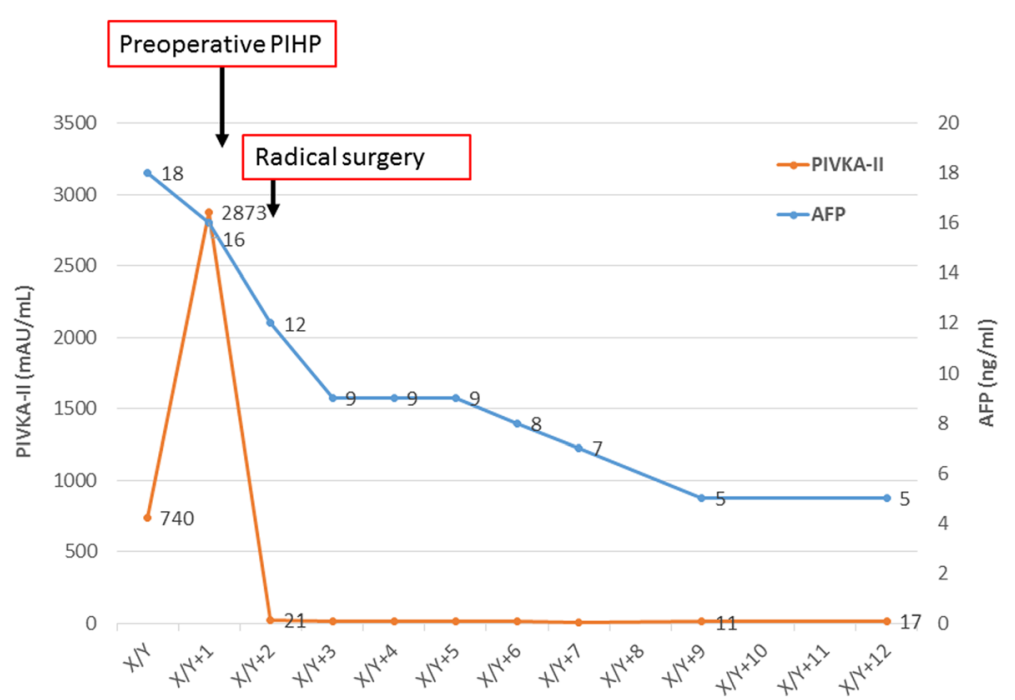

Fig. 4 Perioperative clinical course: After preoperative PIHP, the serum level of AFP and PIVKA-II dramatically declined. She recovered uneventfully after the surgery and remained relapse-free 12 months after the surgery without any postoperative chemotherapy

option. To improve the efficacy of surgical resection for HCC involving PVTT, neoadjuvant/adjuvant therapies were evaluated in several studies [6, 22-24]. Because of the heterogeneous results, the effect of neoadjuvant therapy on the OS was inconclusive.

Since 1989, our center has been using PIHP for the treatment of advanced multiple HCC [6]. In our previous study, the data suggested a significant tumoricidal impact on less sensitive HCC and sufficient sorption with charcoal hemoperfusion of high-dose doxorubicin and MMC regimen [10]. In 2003, we reported the efficacy of reductive surgery plus PIHP for multiple HCC, including HCC with macroscopic PVTT [11]. The favorable survival profile suggested the efficacy of the second PIHP in local tumor control. Based on these previous reports, we extended the use of PIHP as preoperative therapy. In the present case, because the
PVTT in the left portal branch reached the right portal branch and the high level of PIVKA-II indicated high recurrence possibility, surgery was planned after preoperative chemotherapy with PIHP. To the best of our knowledge, this is the first report of a case with pathological complete response after preoperative chemotherapy with PIHP in advanced HCC involving PVTT. Although there were several reports of spontaneous regression of PVTT $[25,26]$, in the present case, we judged PIHP was effective based on the pathohistological assessment.

Recently, the efficacy of preoperative HAI chemotherapy has been shown in patients with HCC and PVTT $[27,28]$. Because PIHP has shown a high objective response rate $(70.6 \%$ complete and partial response) and safety, it might be a better alternative instead of preoperative therapy.

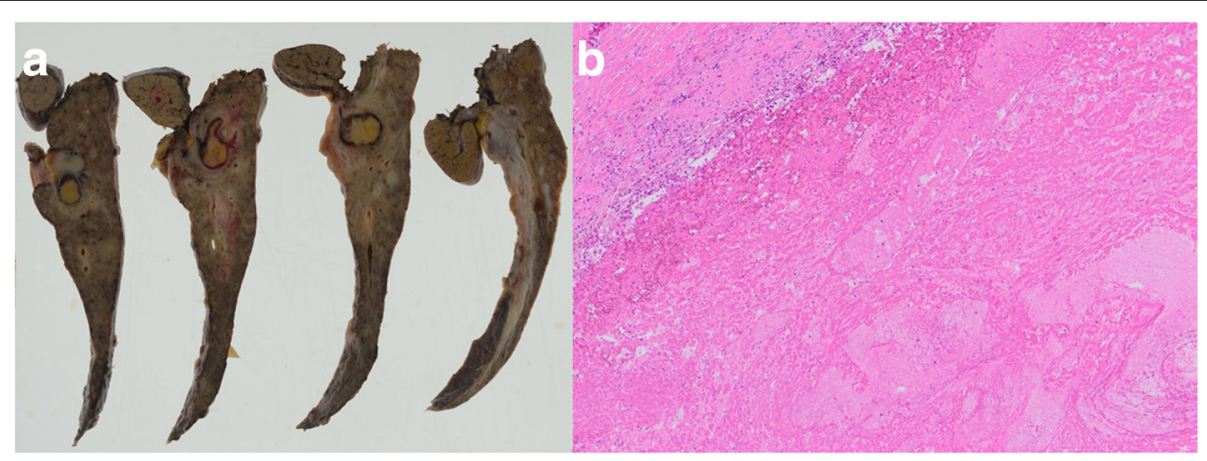

Fig. 5 Macroscopic (a) and microscopic (b) findings on the resected specimen after PIHP: No residual tumor was found at the original site of the HCC, which extended into the portal branch in the left lobe of the liver. Instead, it was completely replaced by granulated and fibrous tissue. Microscopic findings in non-cancerous lesion showed grade 1 inflammation and stage $4 / 4$ fibrosis with histopathologic features of chronic HCV. (hematoxylin and eosin stain, $\times 20$ ) 
The clinical question of whether preoperative or postoperative PIHP is better should be addressed. Preoperative PIHP might not only reduce post-curative therapy recurrence but also downstage tumors from an unresectable to a resectable stage.

\section{Conclusions}

In conclusion, further investigation into surgery following neoadjuvant chemotherapy as a possible therapeutic option for HCC involving PVTT is warranted. PIHP with doxorubicin plus mitomycin $\mathrm{C}$ is a feasible and promising preoperative chemotherapy for HCC involving PVTT.

\section{Consent}

Written informed consent was obtained from the patient for publication of this case report and any accompanying images. A copy of the written consent is available for review by the Editor-in-Chief of this journal.

\section{Abbreviations}

AFP, alpha-fetoprotein; ALT, alanine aminotransferase; AST, aspartate aminotransferase; BCLC, the Barcelona Clinic Liver Cancer; CT, computed tomography; HAl, hepatic arterial infusion; HCC, hepatocellular carcinoma; ICG R15, indocyanine green 15-min retention test; OS, overall survival; PIHP, percutaneous isolated hepatic perfusion; PIVKA-II, protein induced by vitamin $\mathrm{K}$ absence or antagonist II; PVTT, portal vein tumor thrombosis; TE, treatment efficacy

\section{Competing interests}

The authors declare that they have no competing interests.

\section{Authors' contributions}

KA wrote the manuscript and literature search. TF reviewed and edited the manuscript. TA and YK supervised the study. KA, TF, MT, KK, MK, HK, TM, HT, $\mathrm{SA}$, and TG treated and observed the patient. All authors read and approved the final manuscript.

\section{Ethics approval and consent to participate}

The study protocol about PIHP was approved by the Kobe University Hospital institutional review board.

\section{Acknowledgements}

We would like to thank Editage (www.editage.jp) for the English language editing.

Received: 7 April 2016 Accepted: 21 May 2016

Published online: 26 May 2016

\section{References}

1. Forner A, Llovet JM, Bruix J. Hepatocellular carcinoma. Lancet. 2012;379:1245-55.

2. Pang TC, Lam WW. Surgical management of hepatocellular carcinoma. World J Hepatol. 2015;7:245-52.

3. Quirk M, Kim YH, Saab S, Lee EW. Management of hepatocellular carcinoma with portal vein thrombosis. World J Gastroenterol. 2015;21:3462-71.

4. Kokudo N, Hasegawa K, Akahane M, Igaki H, Izumi N, Ichida T, et al. Evidence-based Clinical Practice Guidelines for Hepatocellular Carcinoma: the Japan Society of Hepatology 2013 update (3rd JSH-HCC Guidelines). Hepatol Res.2015:45:123-27.

5. Ban D, Shimada K, Yamamoto Y, Nara S, Esaki M, Sakamoto Y, et al. Efficacy of a hepatectomy and a tumor thrombectomy for hepatocellular carcinoma with tumor thrombus extending to the main portal vein. J Gastrointest Surg. 2009;13:1921-8.

6. Minagawa M, Makuuchi M, Takayama T, Ohtomo K. Selection criteria for hepatectomy in patients with hepatocellular carcinoma and portal vein tumor thrombus. Ann Surg. 2001;233:379-84.
7. Pawlik TM, Poon RT, Abdalla EK, Ikai I, Nagorney DM, Belghiti J, et al. Hepatectomy for hepatocellular carcinoma with major portal or hepatic vein invasion: results of a multicenter study. Surgery. 2005;137:403-10.

8. Wu CC, Hsieh SR, Chen JT, Ho WL, Lin MC, Yeh DC, et al. An appraisal of liver and portal vein resection for hepatocellular carcinoma with tumor thrombi extending to portal bifurcation. Arch Surg. 2000;135:1273-9.

9. Ohkubo T, Yamamoto J, Sugawara Y, Shimada K, Yamasaki S, Makuuchi M, et al. Surgical results for hepatocellular carcinoma with macroscopic portal vein tumor thrombosis. J Am Coll Surg. 2000;191:657-60.

10. Ku Y, Iwasaki T, Fukumoto T, Tominaga M, Muramatsu S, Kusunoki N, et al. Induction of long-term remission in advanced hepatocellular carcinoma with percutaneous isolated liver chemoperfusion. Ann Surg. 1998;227:519-26.

11. Ku Y, Iwasaki T, Tominaga M, Fukumoto T, Takahashi T, Kido M, et al. Reductive surgery plus percutaneous isolated hepatic perfusion for multiple advanced hepatocellular carcinoma. Ann Surg. 2004;239:53-60.

12. Fukumoto T, Tominaga M, Kido M, Takebe A, Tanaka M, Kuramitsu K, et al. Long-term outcomes and prognostic factors with reductive hepatectomy and sequential percutaneous isolated hepatic perfusion for multiple bilobar hepatocellular carcinoma. Ann Surg Oncol. 2014;21:971-8.

13. Kudo M, Kitano M, Sakurai T, Nishida N. General rules for the clinical and pathological study of primary liver cancer, nationwide follow-up survey and clinical practice guidelines: the outstanding achievements of the Liver Cancer Study Group of Japan. Dig Dis. 2015;33:765-70.

14. Available Online: http://www.jcog.jp/doctor/tool/CTCAEv4J_20130409.pdf. Published: 28 May 2009 (v4.03: Jun. 14, 2010).

15. Ikai I, Hatano E, Hasegawa S, Fujii H, Taura K, Uyama N, et al. Prognostic index for patients with hepatocellular carcinoma combined with tumor thrombosis in the major portal vein. J Am Coll Surg. 2006;202:431-8.

16. Villa E, Moles A, Ferretti I, Buttafoco P, Grottola A, Del Buono M, et al. Natural history of inoperable hepatocellular carcinoma: estrogen receptors' status in the tumor is the strongest prognostic factor for survival. Hepatology. 2000;32:233-8.

17. Llovet JM, Bustamante J, Castells A, Vilana R, Ayuso Mdel C, Sala M, et al. Natural history of untreated nonsurgical hepatocellular carcinoma: rationale for the design and evaluation of therapeutic trials. Hepatology. 1999;29:62-7.

18. Ikai I, Yamaoka Y, Yamamoto Y, Ozaki N, Sakai Y, Satoh S, et al. Surgical intervention for patients with stage IV-A hepatocellular carcinoma without lymph node metastasis: proposal as a standard therapy. Ann Surg. 1998;227:433-9.

19. Shimada M, Takenaka K, Kawahara N, Kajiyama K, Yamamoto K, Shirabe K, et al. Surgical treatment strategy for patients with stage IV hepatocellular carcinoma. Surgery. 1996;119:517-22.

20. Forner A, Reig ME, de Lope CR, Bruix J. Current strategy for staging and treatment: the BCLC update and future prospects. Semin Liver Dis. 2010;30:61-74.

21. Shi J, Lai EC, Li N, Guo WX, Xue J, Lau WY, et al. Surgical treatment of hepatocellular carcinoma with portal vein tumor thrombus. Ann Surg Oncol. 2010;17:2073-80.

22. Li Q, Wang J, Sun Y, Cui YL, Juzi JT, Li HX, et al. Efficacy of postoperative transarterial chemoembolization and portal vein chemotherapy for patients with hepatocellular carcinoma complicated by portal vein tumor thrombosis-a randomized study. World J Surg. 2006;30:2004-11. discussion 2012-2003.

23. Yeh ML, Huang Cl, Huang CF, Hsieh MY, Huang JF, Dai CY, et al. Neoadjuvant transcatheter arterial chemoembolization does not provide survival benefit compared to curative therapy alone in single hepatocellular carcinoma. Kaohsiung J Med Sci. 2015;31:77-82.

24. Chua TC, Liauw W, Saxena A, Chu F, Glenn D, Chai A, et al. Systematic review of neoadjuvant transarterial chemoembolization for resectable hepatocellular carcinoma. Liver Int. 2010:30:166-74.

25. Okano A, Takakuwa H, Nakamura T. Spontaneous regression of diffuse intrahepatic recurrence with portal vein tumor thrombus after resection of hepatocellular carcinoma. Clin J Gastroenterol. 2011;4:43-8.

26. Uenishi T, Hirohashi K, Tanaka H, Ikebe T, Kinoshita H. Spontaneous regression of a large hepatocellular carcinoma with portal vein tumor thrombi: report of a case. Surg Today. 2000;30:82-5.

27. Taguchi M, Sakuma Y, Sasanuma H, Sata N, Lefor AK, Sasaki T, et al. Complete pathological response to transcatheter arterial infusion despite a rapidly progressing recurrent hepatocellular carcinoma with portal vein tumor thrombus: a case report. Int J Surg Case Rep. 2015;10:20-4.

28. Kim YG, Eun JR, Kim TN, Lee HJ, Kim JW, Chang JC, et al. Pathological complete remission of advanced hepatocellular carcinoma with main portal vein tumor thrombosis by hepatic arterial infusion chemotherapy. Gut Liver. 2010;4:266-9. 